\title{
Adjunctive subgingival application of Chlorhexidine gel in nonsurgical periodontal treatment for chronic periodontitis: a systematic review and meta-analysis
}

Han Zhao ${ }^{1,2+}$, Jingchao $\mathrm{Hu}^{2,3+}$ and Li Zhao ${ }^{4,5,6^{*}}$ (D)

\begin{abstract}
Background: Subgingival applications of chlorhexidine (CHX) gel are commonly used as an adjunct in nonsurgical periodontal treatment (NSPT) for chronic periodontitis (CP). However, there is lack of systematic review and metaanalysis justifying the effects of adjunctive CHX gel on clinical outcomes. The objective of this meta-analysis was to evaluate the efficacy of adjunctive subgingival administration of CHX gel in NSPT compared to NSPT alone for CP.

Methods: An electronic search of four databases and a manual search of four journals were conducted up to August 2019. Only randomized controlled trials reporting on the clinical outcomes of subgingival use of CHX gel adjunct to scaling and root planing (SRP), as compared to SRP alone or with placebo, for at least 3 months were included. Primary outcomes were probing pocket depth (PPD) reduction and clinical attachment level (CAL) gain at 3 and 6 months, when data on at least three studies were obtained.

Results: Seventeen studies were included for qualitative analysis and seven studies for quantitative analysis (four studies for the application of CHX gel adjunct to SRP at selected sites with at least pocket depth $\geq 4 \mathrm{~mm}$ and three studies for comparison of full-mouth disinfection (FMD) with subgingival use of $\mathrm{CHX}$ gel and full-mouth scaling and root planing (FMSRP). For subgroups, the clinical outcomes between adjunctive use of Xanthan-based CHX gel (XAN-CHX gel) and CHX gel were analyzed. Results indicated a significant improvement of PPD reduction following local adjunctive administration of XAN-CHX gel for SRP at selected sites (MD: $0.15 \mathrm{~mm}$ ). However, no difference was found in CAL gain. Moreover, no significant difference was observed in PPD and CAL at both 3 and 6 months posttreatment between FMD and FMSRP.
\end{abstract}

Conclusion: Adjunctive subgingival administration of XAN-CHX gel at individual selected sites in NSPT appears to provide slight benefits in PPD reduction compared to NSPT alone for CP. Due to the lack of high-quality studies, further studies with larger sample sizes and strict standards are needed to confirm the conclusions.

Keywords: Chronic periodontitis, Chlorhexidine, Subgingival irrigation, Root planing; meta-analysis

\footnotetext{
* Correspondence: lizhao2010@hospital.cqmu.edu.cn

${ }^{+}$Han Zhao and Jingchao Hu contributed equally to this work.

${ }^{4}$ Department of Prosthodontics, Stomatological Hospital of Chongqing

Medical University, Chongqing 400015, China

${ }^{5}$ Chongqing Key Laboratory of Oral Diseases and Biomedical Sciences,

Chongqing 400015, China

Full list of author information is available at the end of the article
}

(c) The Author(s). 2020 Open Access This article is distributed under the terms of the Creative Commons Attribution 4.0 International License (http://creativecommons.org/licenses/by/4.0/), which permits unrestricted use, distribution, and reproduction in any medium, provided you give appropriate credit to the original author(s) and the source, provide a link to the Creative Commons license, and indicate if changes were made. The Creative Commons Public Domain Dedication waiver (http://creativecommons.org/publicdomain/zero/1.0/) applies to the data made available in this article, unless otherwise stated. 


\section{Background}

Chronic periodontitis (CP) is characterized as a complex progressive chronic inflammatory process, which leads to the destruction of periodontal supportive tissue and a further loss of teeth. CP occurs when the magnitude effects of the pathogenic microbial load in the periodontal pocket are larger than that of the hosts immune response $[1,2]$. The basis of periodontal treatment is elimination or suppression of periodontal pathogens. The golden standard of which is mechanical debridement by scaling and root planing (SRP). However, large limitations of physical treatment have been observed due to the difficulty of accessing deep periodontal defects, which compromises the effectiveness of biofilm removal. The persistence of periodontal pathogens, such as Aggregatibacteractinomycetemcomitans and Porphyromonas gingivalis (P.g), were often found following SRP and can result in microbial re-colonization and the consequent destruction of periodontal tissue [3-6]. In regards to this issue, adjunctive systemic and localized antibiotics have been applied to compensate for the limitation of mechanical therapy. Despite the rapid development of a variety of adjunctive local periodontal treatments in recent years, such as metformin, antioxidants, photodynamic treatment and so on [7-9], chlorhexidine (CHX) remains one of the most effective local antimicrobial agents, and is widely used for the local treatment of periodontitis [10-13]. Through the rapid attraction of the negatively charged bacterial cell surface to the cationic $\mathrm{CHX}$ molecule, $\mathrm{CHX}$ shows strong antibacterial activity in the periodontal pocket, along with a lack of toxicity, incompliance from patients and an emergence of resistance microorganisms. However, the high clearance of CHX from the periodontal pocket leads to subtherapeutic CHX concentrations in the local environment after only a short time of subgingival CHX application [14], which results in an insufficient treatment effectiveness $[1,15]$. Given this limitation, CHX Gel with CHX concentration up to 15 times than liquid carriers was developed for periodontal treatment. In recent years, numerous of studies have reported the effectiveness of adjunctive $\mathrm{CHX}$ to nonsurgical periodontal treatment (NSPT). However, contrary results were presented [1013], there is still no consensus on this issue. So far, only one systematic review without quantitative analysis indicated that the positive effect of local subgingival application of CHX Gel adjunctive to NSPT could be not justified as compared to NSPT alone [16]. Therefore, there is lack of strong evidence for support the beneficial effect of subgingival use CHX as adjunct to NSPT.

Full-mouth disinfection (FMD) was proposed by Quirynen in 1995, with the aim of eradicating periodontal pathogens in a short time from all the oropharyngeal habitats (mucous membranes, tongue, tonsils and saliva)
[17]. CHX gel as an adjunct was used in the FMD protocol, which was described as full-mouth scaling and root planing (FMSRP) in 1-2 sessions within $24 \mathrm{~h}$ combined with full-mouth subgingival irrigation with $\mathrm{CHX}$ gel, as well as a tongue brush and mouthwash by means of CHX [2, 17-20]. However, whether the use of antiseptics played a role in FMD is still unclear.

The aim of this systematic review and meta-analysis was to evaluate the benefits of a subgingival administration of CHX gel as an adjunct to NSPT for the treatment of $\mathrm{CP}$.

\section{Method \\ Focus questions}

Whether subgingival chlorhexidine gel application as an adjunct to nonsurgical periodontal treatment provides additional benefit to clinical outcomes in chronic periodontitis?

\section{Search strategy}

The review and meta-analysis were based on the Preferred Reporting Items for Meta-Analysis (PRISMA) statement [21]. Three reviewers ( $\mathrm{HZ}, \mathrm{JCH}$ and LZ) conducted an independent search of three databases, including PubMed, EMBASE and the Cochrane Collaboration Library on the 20 August 2019 for articles addressing the focused question. Furthermore, a search of the Open Grey database was performed, and a hand search was conducted of following journals: Journal of Dental Research, Journal of Periodontology, Journal of Clinical Periodontology and Journal of Periodontal Research from 2000 until 2019.

\section{Study selection}

Titles and abstracts were reviewed for eligibility by two independent reviewers $(\mathrm{HZ}, \mathrm{JCH})$ according to the inclusion criteria. Studies that met all inclusion criteria or met some of inclusion criteria but did not meet any of the exclusion criteria were admitted for full-text review. In this phase, full-text papers were assessed in line with the exclusion criteria. And the reasons for exclusion were recorded (Additional file 1: Table S1). Any disagreements were resolved on discussion between the three reviewers and a consensus was reached through voting. The agreement value between the reviewers was calculated using Kappa statistics, which is used to measure inter-rater reliability. The classification of Kappa Value was suggested: $\leq 0$ as indicating no agreement, $0.01-0.20$ as none to slight, $0.21-0.40$ as fair, $0.41-0.60$ as moderate, $0.61-0.80$ as substantial, and $0.81-1.00$ as perfect agreement [22].

The search strategy for PubMed (adapted to the other databases) is listed below: 
(periodontitis OR periodontal disease) AND ((((chlorhexidine, OR chlorhexidine gluconate, OR xanthan OR xanthan chlorhexidine) AND gel) AND (subgingival, OR subgingival curettage, OR dental scaling, OR root planing OR dental prophylaxis)) OR full mouth disinfection)

\section{Primary and secondary outcomes}

The primary outcomes were probing pocket depth (PPD) reduction and the clinical attachment level (CAL) gain at 3 and 6 months post-therapy. The secondary outcome was adverse events.

\section{Eligibility criteria}

The inclusion criteria for the studies were: 1) randomized controlled trials (RCTs); 2) comparison of SRP alone/placebo and CHX gel adjunct to SRP; or comparison of FMSRP alone/placebo and FMD, including subgingival use of $\mathrm{CHX}$ gel; 3) follow-up of at least 3 months; 4) reported data on clinical parameters (CAL and PPD) and 5) publication in English only.

The exclusion criteria were: 1) not RCTs; 2) duplicate publications; 3) Inadequate treatment strategy: $\mathrm{CHX}$ was used as mono-therapy or $\mathrm{CHX}$ was used as adjunct to surgery or other treatment; 4) follow-up less than 3 months; 5) reported only microbiological findings with no reference to clinical result; 6) not in English.

\section{Quality assessment}

The methodology quality of the included articles was evaluated independently by two reviewers ( $\mathrm{HZ}$ and $\mathrm{JCH})$ based on recommendations from the CONSORT statement [23]. Quality assessments of the included studies were conducted using the revised risk of bias assessment tool from the Cochrane Collaboration's handbook version 5.2.0 [24], which includes seven criteria: random sequence generation; allocation concealment; blinding of participants and personnel; blinding of outcome assessment; incomplete outcome data; selective reporting and other sources of bias. Each category was estimated on whether it could impact the overall results and was further qualified as either low, high or unclear. Overall, each article was judged as (i) low risk of bias, (ii) unclear risk of bias or (iii) high risk of bias. When a trial did not meet all four criteria for randomization and blinding methods, it was excluded from quantitative analysis, as its low quality and high bias may have subverted the validity of the results and conclusions.

\section{Data collection process/data items}

Data of each included study were recorded using a standardized data extraction form, including study design, number of patients, demographics, inclusion criteria, types of $\mathrm{CHX}$ gel, timing and frequency of $\mathrm{CHX}$ gel application, number of adverse events and length of follow-up.

\section{Data synthesis}

The meta-analysis was performed using RevMan version 5.3 (2014). Mean differences (MD) with 95\% confidence intervals $(95 \% \mathrm{CI})$ were used for continuous data. The I2 value was used to access the statistical heterogeneity of the studies. If the heterogeneity was evaluated as $\mathrm{I} 2 \leq$ $50 \%$, a fixed effects model was applied. When the heterogeneity was assessed as $\mathrm{I} 2>50 \%$, a random effects model was used. The inverse-variance method performed, and the overall effect was defined as statistically significant if the $p$ value $<0.05$.The I 2 value was classified into four levels: i) no heterogeneity, between 0 and $25 \%$; ii) low heterogeneity, $25-50 \%$; iii) moderate heterogeneity, $50-75 \%$ and iiii) high heterogeneity, $75-100 \%$ [25].

\section{Results \\ Study selection}

In the initial search, a total of 487 studies were identified; 171 PubMed, 166 Embase and 139 from the Cochrane Library database. Three papers were found through the Open Grey search and eight papers were selected following a manual search. After removal of duplicates $(n=221), 266$ papers were included in the selection phase of titles and abstracts. A total of 240 articles were excluded, and 27 papers were selected for fulltext reading. In this phase, 10 studies were further excluded (Additional file 1: Table S1) and 17 papers were finally included in the qualitative analysis $[2,10-12,17-$ 19, 26-35]., The kappa value for inter-reviewer agreement was 0.92 indicating high degree of inter-rater reliability. Figure 1 shows the study identification flowchart based PRISMA19 with the reasons for exclusion.

\section{Description of the included studies}

Seventeen articles met the criteria and were included for qualitative analysis. Thirteen studies reported subgingival application of CHX gel adjunct to SRP at selected sites with a moderate to deep probing depth (at least 4 $\mathrm{mm}$ in all studies) [8-10, 25-34]. Nine studies were split-mouth RCTs [10, 12, 27-29, 32-35], four were parallel RCTs $[11,26,30,31]$ and three studies used a placebo in the control $[12,31,33]$. From the 13 papers, 10 showed the clinical outcomes of adjunctive subgingival delivered Xanthan-based CHX gel (XAN-CHX gel) in SRP and SRP alone [26-35]; CHX concentration in the XAN-CHX gel was $1.5 \%$ in nine studies and $2.5 \%$ in one study [34], and another three studies reported the use of gels containing $0.5,1$ and $2 \% \mathrm{CHX}$ without Xanthan gum [10-12]. Patient samples ranged from five to 98 . One included study compared the clinical outcomes 


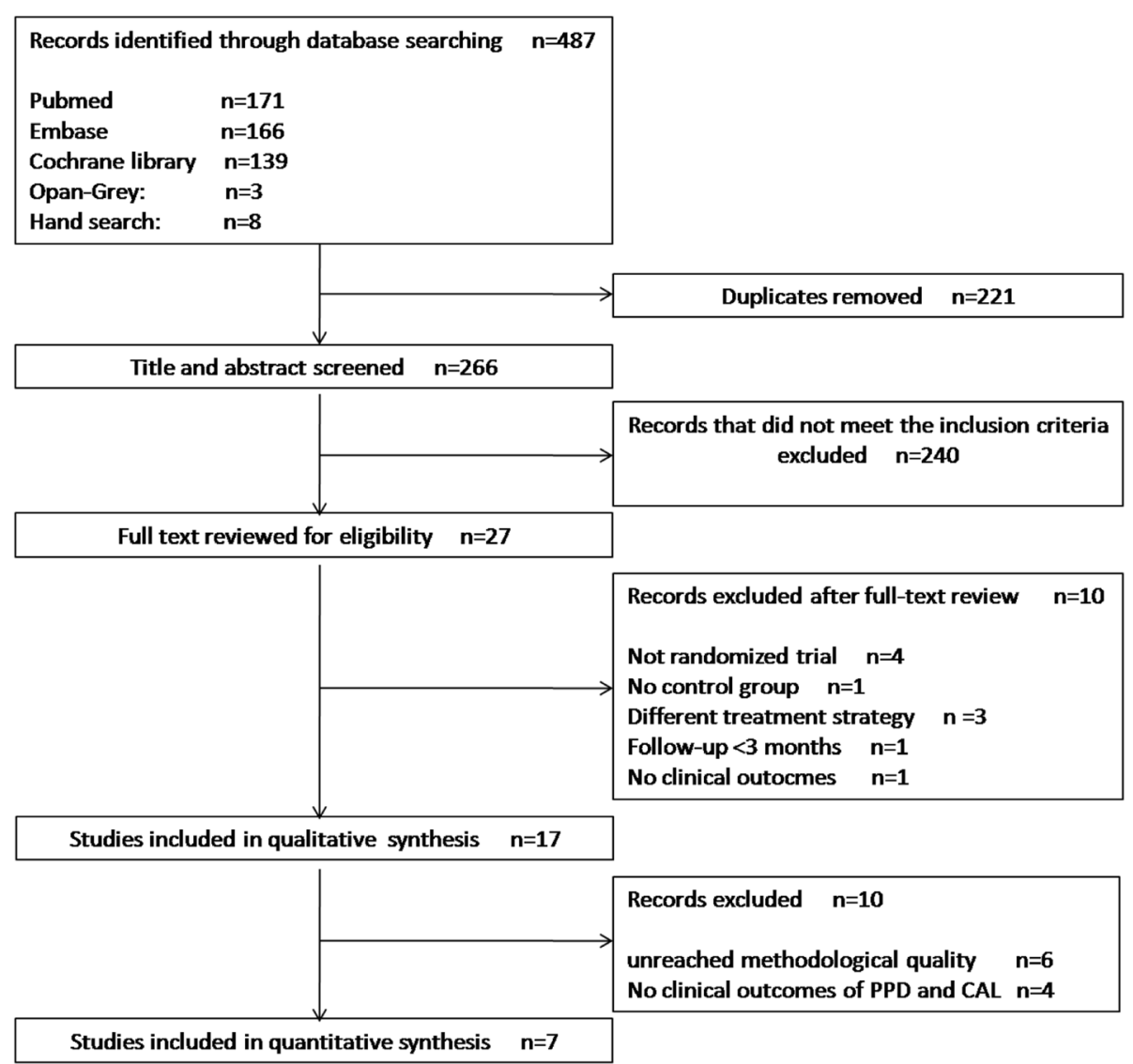

Fig. 1 Flow chart of the study identification based PRISMA19 with the reasons for exclusion

between SRP plus XAN-CHX gel and SRP alone for patients with diabetes mellitus type 2 [27]. The timing and frequency of $\mathrm{CHX}$ Gel application varied between the trials. In all 13 studies but four [10, 12, 27, 32], the CHX gel was applied once at baseline after SRP. In the other four studies, the application of CHX gel was described as three times at baseline, 10 day and 20 day follow-ups [27], once at 1 month after treatment [31] and three times within $10 \mathrm{~min}$ at baseline $[10,12]$. The follow-ups ranged from 1 month to 6 months after SRP.

An additional arm of the four studies evaluated the results between FMD and FMSRP [2, 17-19]. All studies were RCTs, and one used a placebo gel and solution in the FMSRP group. The number of participants ranged from 18 to 38 . Follow-ups varied from 1 month to 12 months. One study included patients with diabetes mellitus type 2 [18]. A 1\% CHX gel was used in all of the trials. The timing and frequency variations for the $\mathrm{CHX}$ gel ranged from once at baseline $[17,19]$ and three times in $10 \mathrm{~min}$ at baseline [18] to three times within $10 \mathrm{~min}$ at first session, second session of FMSRP and at 1 week of follow-up, respectively [2]. Table 1 shows the summary of the characteristics of the included studies.

\section{Risk of bias assessment}

All studies were RCTs. Seven studies did not report on their randomization and allocation methods in detail [11, $12,27,28,30,32,34,35]$, and from these, six studies also did not describe the blinding methods of participants and personnel as well as their assessment $[11,27,28,30,32$, 35], continued $\mathrm{CHX}$ rinsing stains the tooth and tongue surfaces, examiners could deduce which subjects were receiving CHX though these changes, and all the four studies in the analysis for comparison between FMD and FMSRP were considered at most to be single-blinded ${ }^{2,1719}$. Given examiner blinding was performed strictly in three studies, the detection bias for the three articles was qualified as 'unclear' $[2,17,19]$. Overall, for all 17studies, six were assessed to have a low risk of bias $[10,17,18,26,29$, $31]$, two were judged as an unclear risk of bias [12, 33], nine were considered to have a high risk of bias $[2,11,19$, $27,28,30,32,34,35]$, and six were excluded from the quantitative analysis $[11,27,28,30,32,35]$. . The summary of quality assessment is showed in Table 2 .

\section{Synthesis of results}

All 17 studies reported on clinical outcomes with the use of adjunctive CHX Gel. The clinical results of these 
Table 1 Characteristics of included studies. Variables were listed in this systematic review (including:study design, patient demographics, methodology, number of adverse events and length of follow-up). Outcome difference is reported only between adjunctive CHX gel to SRP and SRP alone

\begin{tabular}{|c|c|c|c|c|c|c|c|c|c|c|}
\hline \multirow[t]{2}{*}{ Administration } & \multirow[t]{2}{*}{ Study } & \multirow[t]{2}{*}{ Design } & \multicolumn{4}{|c|}{ Participants } & \multicolumn{2}{|l|}{ Methodology } & \multirow[t]{2}{*}{$\mathrm{AE}$} & \multirow{2}{*}{$\begin{array}{l}\text { Follow- } \\
\text { up (m) }\end{array}$} \\
\hline & & & $\begin{array}{l}\mathrm{N} \\
(\mathrm{C} / \\
\mathrm{T})\end{array}$ & Inclusion criteria & SD & age & $\begin{array}{l}\text { Description } \\
\text { of Gel }\end{array}$ & CHX Gel Application & & \\
\hline \multirow[t]{13}{*}{$\begin{array}{l}\text { Application at } \\
\text { selected sites }\end{array}$} & $\begin{array}{l}\text { Faramarzi M } \\
\text { et al. (2017) } \\
\text { [26] }\end{array}$ & $P$ & $\begin{array}{l}68 \\
(34 / \\
34)\end{array}$ & at least eight teeth with PD 4-8 mm & $\begin{array}{l}2- \\
\mathrm{DM}\end{array}$ & $\begin{array}{l}30- \\
60 \\
\text { years }\end{array}$ & $\begin{array}{l}\text { XAN-CHX } \\
1.5 \% \mathrm{CHX} \\
\text { gel }\end{array}$ & $\begin{array}{l}\text { one time after } 2 \text { nd } \\
\text { SRP (baseline, } 2 \\
\text { week after 1st SRP) }\end{array}$ & / & 3,6 \\
\hline & $\begin{array}{l}\text { Phogat M } \\
\text { et al. (2014) } \\
\text { [27] }\end{array}$ & S & $\begin{array}{l}30 \\
(30 / \\
30)\end{array}$ & $\begin{array}{l}\text { at least } 3 \text { nonadjacent interproximal } \\
\text { sites with PD 4-8 mm }\end{array}$ & no & $\begin{array}{l}30- \\
50 \\
\text { years }\end{array}$ & $\begin{array}{l}\text { XAN-CHX } \\
1.5 \% \mathrm{CHX} \\
\text { gel }\end{array}$ & $\begin{array}{l}\text { one time at baseline, } \\
10 \text { days and } 20 \text { days }\end{array}$ & / & 1,3 \\
\hline & $\begin{array}{l}\text { Jain M et al. } \\
\text { (2013) [28] }\end{array}$ & $S$ & $\begin{array}{l}30 \\
(30 / \\
30)\end{array}$ & $\begin{array}{l}2 \text { sites located on the same side PD } \\
\text { between } 5 \text { to } 7 \mathrm{~mm}\end{array}$ & no & $\begin{array}{l}30- \\
60 \\
\text { years }\end{array}$ & $\begin{array}{l}\text { XAN-CHX } \\
1.5 \% \text { CHX } \\
\text { gel }\end{array}$ & one time at baseline & / & $1.5,3,6$ \\
\hline & $\begin{array}{l}\text { Chitsazi MT } \\
\text { et al. (2013) } \\
\text { [29] }\end{array}$ & S & $\begin{array}{l}20 \\
(20 / \\
20)\end{array}$ & $\begin{array}{l}\text { one site per quadrant with } \mathrm{PD} \geq 4 \\
\mathrm{~mm} \text { and } \mathrm{BOP}(+)\end{array}$ & no & $\begin{array}{l}\text { mean } \\
46.5 \\
\text { years }\end{array}$ & $\begin{array}{l}\text { XAN-CHX } \\
1.5 \% \mathrm{CHX} \\
\text { gel }\end{array}$ & one time at baseline & 0 & 1,3 \\
\hline & $\begin{array}{l}\text { Chauhan AS } \\
\text { et al. (2013) } \\
\text { [30] }\end{array}$ & $P$ & $\begin{array}{l}40 \\
(20 / \\
20)\end{array}$ & at least 8 teeth with PD $4-8 \mathrm{~mm}$ & no & $\begin{array}{l}30- \\
65 \\
\text { years }\end{array}$ & $\begin{array}{l}\text { XAN-CHX } \\
1.5 \% \text { CHX } \\
\text { gel }\end{array}$ & one time at baseline & / & 1,3 \\
\hline & $\begin{array}{l}\text { Matesanz P } \\
\text { et al. (2013) } \\
\text { [31] }\end{array}$ & $\begin{array}{l}\text { P } \\
\text { (placebo) }\end{array}$ & $\begin{array}{l}22 \\
(12 / \\
10)\end{array}$ & $\begin{array}{l}\text { at least } 16 \text { teeth and at least } 3 \text { teeth } \\
\text { per quadrant, } 4-10 \text { pockets with } \\
\text { PPD }>4 \mathrm{~mm} \text { and } \mathrm{BOP}(+) \text {, or at a } \\
\text { programmed supportive visit }\end{array}$ & no & $\begin{array}{l}\text { elder } \\
\text { than } \\
30 \\
\text { years }\end{array}$ & $\begin{array}{l}\text { XAN-CHX } \\
1.5 \% \mathrm{CHX} \\
\text { gel }\end{array}$ & one time at baseline & 0 & $1,3,6$ \\
\hline & $\begin{array}{l}\text { Verma A } \\
\text { et al. (2012) } \\
{[32]}\end{array}$ & S & $\begin{array}{l}46 \\
(46 / \\
46)\end{array}$ & $\begin{array}{l}\text { at least two non-adjacent interproxi- } \\
\text { mal sites with PD5-8 mm and } \mathrm{BOP}(+)\end{array}$ & no & $\begin{array}{l}30- \\
65 \\
\text { years }\end{array}$ & $\begin{array}{l}\text { XAN-CHX } \\
1.5 \% \text { CHX } \\
\text { gel }\end{array}$ & $\begin{array}{l}\text { one time } 1 \text { month } \\
\text { after SRP }\end{array}$ & / & 1,3 \\
\hline & $\begin{array}{l}\text { Kranti K } \\
\text { et al. (2010) } \\
\text { [33] }\end{array}$ & $\begin{array}{l}\text { S } \\
\text { (placebo) }\end{array}$ & $\begin{array}{l}10 \\
(10 / \\
10)\end{array}$ & $\begin{array}{l}\text { at least } 4 \text { periodontal pockets with } \\
\text { PPD } 5-8 \mathrm{~mm}\end{array}$ & yes & $\begin{array}{l}25-65 \\
\text { ears }\end{array}$ & $\begin{array}{l}\text { XAN-CHX } \\
1.5 \% \mathrm{CHX} \\
\text { gel }\end{array}$ & one time at baseline & / & 3,6 \\
\hline & $\begin{array}{l}\text { Paolantonio } \\
\text { M (2009) } \\
{[34]}\end{array}$ & S & $\begin{array}{l}98 \\
(98 / \\
98)\end{array}$ & $\begin{array}{l}\text { at least two teeth with } \mathrm{PD} \geq 5 \mathrm{~mm} \\
\text { and } \mathrm{BOP}(+)\end{array}$ & no & $\begin{array}{l}24- \\
58 \\
\text { years }\end{array}$ & $\begin{array}{l}\text { XAN-CHX } \\
2.5 \% \mathrm{CHX} \\
\text { gel }\end{array}$ & one time at baseline & / & 3,6 \\
\hline & $\begin{array}{l}\text { Gupta R } \\
\text { et al. (2008) } \\
\text { [35] }\end{array}$ & S & $\begin{array}{l}30 \\
(30 / \\
30)\end{array}$ & $\begin{array}{l}\text { at least three teeth, (at least one } \\
\text { tooth apart), with PPD } 5-8 \mathrm{~mm} \text { and } \\
\mathrm{BOP}(+)\end{array}$ & no & $\begin{array}{l}25- \\
75 \\
\text { years }\end{array}$ & $\begin{array}{l}\text { XAN-CHX } \\
1.5 \% \text { CHX } \\
\text { gel }\end{array}$ & one time at baseline & / & 1,3 \\
\hline & $\begin{array}{l}\text { lecic J et al. } \\
\text { (2016) [10] }\end{array}$ & S & $\begin{array}{l}5 \\
(5 / \\
5)\end{array}$ & at least two bilateral PPD $\geq 5 \mathrm{~mm}$ & no & $\begin{array}{l}21- \\
52 \\
\text { years }\end{array}$ & $\begin{array}{l}0.5 \% \mathrm{CHX} \\
\text { gel }\end{array}$ & $\begin{array}{l}\text { three times within } \\
10 \text { min }\end{array}$ & / & 1,3 \\
\hline & $\begin{array}{l}\text { unsal E et al. } \\
\text { (1994) } 11\end{array}$ & $P$ & $\begin{array}{l}15 \\
(8 / \\
7)\end{array}$ & $\begin{array}{l}\text { at least } 3 \text { teeth in each quadrant } \\
\text { with } 2 \text { sites with } P P D \geq 4 \mathrm{~mm} \text { and } \\
\mathrm{BOP}(+)\end{array}$ & no & $\begin{array}{l}30- \\
57 \\
\text { years }\end{array}$ & $1 \%$ CHX gel & One time at baseline & / & 3 \\
\hline & $\begin{array}{l}\text { Oosterwaal } \\
\text { PJM et al. } \\
\text { (1991) [12] }\end{array}$ & $\begin{array}{l}\text { S } \\
\text { (placebo) }\end{array}$ & $\begin{array}{l}10 \\
(10 / \\
10)\end{array}$ & $\begin{array}{l}\text { at least } 4 \text { interdental PPD } 7-9 \mathrm{~mm} \text { in } \\
\text { single rooted teeth and } \mathrm{BOP}(+)\end{array}$ & no & $\begin{array}{l}33- \\
62 \\
\text { years }\end{array}$ & $2 \% \mathrm{CHX}$ gel & $\begin{array}{l}3 \text { times within } 10 \\
\text { min at baseline }\end{array}$ & / & $1,3,6$ \\
\hline \multirow[t]{4}{*}{$\begin{array}{l}\text { Full-mouth } \\
\text { application }\end{array}$} & $\begin{array}{l}\text { Fonseca DC } \\
\text { et al. (2015) } \\
{[17]}\end{array}$ & $P$ & $\begin{array}{l}30 \\
(15 / \\
15)\end{array}$ & $\begin{array}{l}\text { mild to moderate chronic } \\
\text { periodontitis, at least } 18 \text { natural teeth }\end{array}$ & yes & $\begin{array}{l}35- \\
60 \\
\text { years }\end{array}$ & $1 \%$ CHX gel & one time at baseline & / & 3,6 \\
\hline & $\begin{array}{l}\text { Santos VR } \\
\text { et al. (2013) } \\
{[18]}\end{array}$ & $\begin{array}{l}\text { P } \\
\text { (placebo) }\end{array}$ & $\begin{array}{l}37 \\
(18 / \\
19)\end{array}$ & $\begin{array}{l}\text { at least } 15 \text { teeth, } 30 \% \text { of the sites } \\
\text { with concomitant PD and } C A L>4 \\
\text { mm }\end{array}$ & $\begin{array}{l}2- \\
\mathrm{DM}\end{array}$ & $\begin{array}{l}37- \\
75 \\
\text { years }\end{array}$ & $1 \%$ CHX gel & $\begin{array}{l}3 \text { times within } 10 \\
\text { min at baseline }\end{array}$ & $\begin{array}{l}\mathrm{T}: \\
17 \\
\mathrm{C}: \\
12\end{array}$ & $3,6,12$ \\
\hline & $\begin{array}{l}\text { swierkot } \\
\text { et al. (2009) } \\
{[19]}\end{array}$ & $P$ & $\begin{array}{l}18 \\
(9 / \\
9)\end{array}$ & $\begin{array}{l}\text { at least } 20 \text { teeth with at least six sites } \\
\text { PPD } \geq 5 \mathrm{~mm} \text { and } \mathrm{BOP}(+)\end{array}$ & no & $\begin{array}{l}28- \\
63 \\
\text { years }\end{array}$ & $1 \% \mathrm{CHX}$ gel & one time at baseline & 0 & $1,2,4,8$ \\
\hline & $\begin{array}{l}\text { Quirynen M } \\
\text { et al. (2006) }\end{array}$ & $P$ & $\begin{array}{l}28 \\
(14 /\end{array}$ & $\begin{array}{l}\text { at least } 18 \text { teeth, at least } 2 \text { multi- } \\
\text { rooted and/or } 2 \text { single-rooted teeth }\end{array}$ & no & $\begin{array}{l}30- \\
75\end{array}$ & $1 \% \mathrm{CHX}$ gel & $\begin{array}{l}\text { three times within } \\
10 \text { min at first }\end{array}$ & / & $2,4,8$ \\
\hline
\end{tabular}


Table 1 Characteristics of included studies. Variables were listed in this systematic review (including:study design, patient demographics, methodology, number of adverse events and length of follow-up). Outcome difference is reported only between adjunctive CHX gel to SRP and SRP alone (Continued)

\begin{tabular}{|c|c|c|c|c|c|c|c|c|c|c|}
\hline \multirow[t]{2}{*}{ Administration } & \multirow[t]{2}{*}{ Study } & \multirow[t]{2}{*}{ Design } & \multicolumn{4}{|c|}{ Participants } & \multicolumn{2}{|c|}{ Methodology } & \multirow[t]{2}{*}{$\mathrm{AE}$} & \multirow{2}{*}{$\begin{array}{l}\text { Follow- } \\
\text { up (m) }\end{array}$} \\
\hline & & & $\begin{array}{l}\mathrm{N} \\
(\mathrm{C} / \\
\mathrm{T})\end{array}$ & Inclusion criteria & $\mathrm{SD}$ & age & $\begin{array}{l}\text { Description } \\
\text { of Gel }\end{array}$ & CHX Gel Application & & \\
\hline & {$[2]$} & & 14) & $\begin{array}{l}\text { in the first quadrant, at least } 6 \text { sites } \\
\text { PPD } 6 \mathrm{~mm} \text {, radiographic bone } \\
\text { loss } \geq 25 \%\end{array}$ & & years & & $\begin{array}{l}\text { sessecion, second } \\
\text { session, and 1-week } \\
\text { follow-up }\end{array}$ & & \\
\hline
\end{tabular}

Studies varied according to the design type of studies, the inclusion or exclusion of patients with systemic disease, different concentration and composition of chlorhexidine gel and different timing and frequency of $\mathrm{CHX}$ gel application. Adverse events and follow-up period were recorded

$P$ Intersubject parallel study, $S$ Intrasubject split-mouth study, $N$ Number, $T$ Test group, $C$ Control group, SD Systemic disease, 2-DM Diabetes mellitus type 2, XAN

Xanthan gum, $\mathrm{CHX}$ Chlorhexidine, XAN-CHX Xanthan-based chlorhexidine, min Minutes, AE Adverse events, $m$ Month/months

Table 2 Risk of bias assessment

\begin{tabular}{|c|c|c|c|c|c|c|c|c|c|}
\hline Author (year) & $\begin{array}{l}\text { Random } \\
\text { sequence } \\
\text { generation }\end{array}$ & $\begin{array}{l}\text { Allocation } \\
\text { concealment }\end{array}$ & $\begin{array}{l}\text { Blinding of } \\
\text { participants and } \\
\text { personnel }\end{array}$ & $\begin{array}{l}\text { Blinding of } \\
\text { outcome } \\
\text { assessment }\end{array}$ & $\begin{array}{l}\text { Incomplete } \\
\text { outcome data }\end{array}$ & $\begin{array}{l}\text { Selective } \\
\text { reporting }\end{array}$ & $\begin{array}{l}\text { Other } \\
\text { bias }\end{array}$ & $\begin{array}{l}\text { Risk of } \\
\text { bias }\end{array}$ & \\
\hline $\begin{array}{l}\text { Faramarzi M } \\
\text { et al. (2017) [26] }\end{array}$ & $\circ$ & $\circ$ & $?$ & $\circ$ & $\circ$ & $\circ$ & $\circ$ & low & \\
\hline $\begin{array}{l}\text { Phogat M et al. } \\
\text { (2014) [27] }\end{array}$ & $?$ & $?$ & $x$ & $x$ & $?$ & $?$ & $\circ$ & high & exclusion \\
\hline $\begin{array}{l}\text { Jain M et al. } \\
\text { (2013) [28] }\end{array}$ & $?$ & $?$ & $x$ & $x$ & $\circ$ & $?$ & $\circ$ & high & exclusion \\
\hline $\begin{array}{l}\text { Chitsazi MT } \\
\text { et al. (2013) [29] }\end{array}$ & $\circ$ & $\circ$ & $?$ & $\circ$ & $\circ$ & $\circ$ & $\circ$ & low & \\
\hline $\begin{array}{l}\text { Chauhan AS } \\
\text { et al. (2013) [30] }\end{array}$ & $?$ & $?$ & $x$ & $x$ & $\circ$ & $?$ & $\circ$ & high & exclusion \\
\hline $\begin{array}{l}\text { Verma A et al. } \\
\text { (2012) [32] }\end{array}$ & $?$ & $?$ & $x$ & $x$ & $?$ & $?$ & $\circ$ & high & exclusion \\
\hline $\begin{array}{l}\text { Matesanz P } \\
\text { et al. (2013) [31] }\end{array}$ & $\circ$ & $\circ$ & $\circ$ & $\circ$ & $\circ$ & $\circ$ & $\circ$ & low & \\
\hline $\begin{array}{l}\text { Kranti K et al. } \\
\text { (2010) [33] }\end{array}$ & $\circ$ & $\circ$ & $\circ$ & $\circ$ & $?$ & $?$ & $\circ$ & unclear & \\
\hline $\begin{array}{l}\text { Paolantonio M. } \\
\text { (2009) [34] }\end{array}$ & $\circ$ & $?$ & $x$ & $\circ$ & $\circ$ & $\circ$ & $\circ$ & high & \\
\hline $\begin{array}{l}\text { Gupta R et al. } \\
\text { (2008) [35] }\end{array}$ & $?$ & $?$ & $x$ & $x$ & $?$ & $?$ & $\circ$ & high & exclusion \\
\hline $\begin{array}{l}\text { Fonseca DC } \\
2015 \text { [17] }\end{array}$ & $\circ$ & $\circ$ & $\circ$ & $?$ & $\circ$ & $\circ$ & $\circ$ & low & \\
\hline $\begin{array}{l}\text { santos VR } 2013 \\
\text { [18] }\end{array}$ & $\circ$ & $\circ$ & $\circ$ & $?$ & $\circ$ & $\circ$ & $\circ$ & low & \\
\hline $\begin{array}{l}\text { Quirynen M } \\
2006 \text { [2] }\end{array}$ & $\circ$ & $\circ$ & $x$ & $?$ & $\circ$ & $\circ$ & $\circ$ & high & \\
\hline $\begin{array}{l}\text { Swierkot K } 2009 \\
\text { [19] }\end{array}$ & $\circ$ & $\circ$ & $?$ & $x$ & $\circ$ & $\circ$ & $\circ$ & high & \\
\hline $\begin{array}{l}\text { unsal E } 1994 \\
\text { [11] }\end{array}$ & $?$ & $?$ & $x$ & $x$ & $\circ$ & $\circ$ & $\circ$ & high & exclusion \\
\hline lecic J 2016 [10] & $\circ$ & $\circ$ & $?$ & $\circ$ & $\circ$ & $\circ$ & $\circ$ & low & \\
\hline $\begin{array}{l}\text { Oosterwaal PJM } \\
1991 \text { [12] }\end{array}$ & $\circ$ & $?$ & $\circ$ & $\circ$ & $?$ & $\circ$ & $\circ$ & unclear & \\
\hline
\end{tabular}

०: low risk of bias;?: unclear risk of bias; $x$ : high risk of bias;

Exclusion: when a trial did not meet all four criteria for randomization and blinding methods, it was excluded from quantitative analysis, as its low quality and high bias may have subverted the validity of the results and conclusions 
studies are summarized in Additional file 2: Table S2. There was no consensus on the clinical efficacy of adjunctive CHX gel to SRP at selected sites. A significant improvement in PPD and/or CAL was reported in a number of studies using XAN-CHX gel [27, 28, 30, 3235]. Whereas, several studies showed no additional benefit in clinical outcomes with the adjunctive use of CHX gel $[10-12,26,29,31]$. In addition, all three studies using CHX gels that did not contain Xanthan gum reported no clinical benefits in the test group [10-12]. For comparing FMD and FMSRP, one study showed a significant improvement of PPD at 6 months [17]. In the other three studies, no sufficient evidence supported that FMD provided any significant improved clinical outcomes in terms of PPD and CAL $[2,18,19]$.

Quantitative analysis was performed when data on at least three studies at 3 and/or 6 months follow-up $( \pm 2$ months) was obtained. Six trials were excluded because of an unreached methodological quality for the requirement of this meta-analysis. Four trials were not included in the quantitative synthesis due to a lack of clinical outcomes in terms of PPD and CAL at follow-up [2, 12, 33, 34]. Finally, four studies were included for the quantitative analysis of subgingival application of $\mathrm{CHX}$ gel at selected sites in terms of PPD reduction and CAL gain $^{10,26,29,31}$, three studies were included for analysis of full-mouth subgingival application of $\mathrm{CHX}$ gel in terms of the mean PPD and mean CAL at 3-4 and 6-8 months [17-19]. Four trials reported the adverse events after treatment $[18,19,29,32]$. Changes in PPD and CAL at selected sites 6 months after CHX gel administration and the mean bleeding of probing (BOP) value at followups after treatment were not conducted due to a lack of data available in the meta-analysis.

\section{Pooled outcomes}

For the adjunctive application of CHX gel to SRP compared to SRP alone at selected sites, the meta-analysis showed a significant improvement in PPD reduction, with a mean MD of $0.15 \mathrm{~mm}$ (MD: 0.15 [95\% CI: 0.040.25 ]; $p=0.005$ ), no heterogeneity was observed among the studies $(\mathrm{I} 2=0 \%)$ (Fig. 2a); No significant differences were found on the CAL gain between the groups (MD: 0.03 [95\% CI: $-0.09-0.15$ ]; $p=0.09$ ) and moderate heterogeneity was indicated $(\mathrm{I} 2=54 \%$ ) (Fig. $2 \mathrm{~b})$. For subgroup analysis, adjunctive XAN-CHX gel provided a significant PPD reduction, with a MD of $0.15 \mathrm{~mm}$ with no heterogeneity (MD: 0.15 [95\% CI: 0.04-0.25]; $p=$ $0.005, \mathrm{I} 2=11 \%$ ) (Fig. 3a). Whereas, no additional benefit for CAL gain was showed in the XAN-CHX group with a low heterogeneity among the studies (MD: 0.05 [95\% CI: $-0.05-0.15] ; p=0.33, \mathrm{I} 2=50 \%$ ) (Fig. 3b).

For full-mouth use of CHX gel, both the mean PPD and CAL showed no significant differences at 3-4 and 6-8 months. The overall effect size for PPD was -0.18 $\mathrm{mm}$ at $3-4$ months and $-0.12 \mathrm{~mm}$ at $6-8$ months, and a high heterogeneity was observed among the studies [34 months (MD: -0.43 [95\% CI: $-0.63-0.27] ; p=0.43$, $\mathrm{I} 2=76 \%$ ) (Fig. 4a), 6-8 months ( 0.12 [95\% CI: $-0.58-$ 0.35 ]; $p=0.62$, I2 $=78 \%$ ) (Fig. 4b)]. CAL was $0.09 \mathrm{~mm}$ at

\section{(a) \\ (b)}

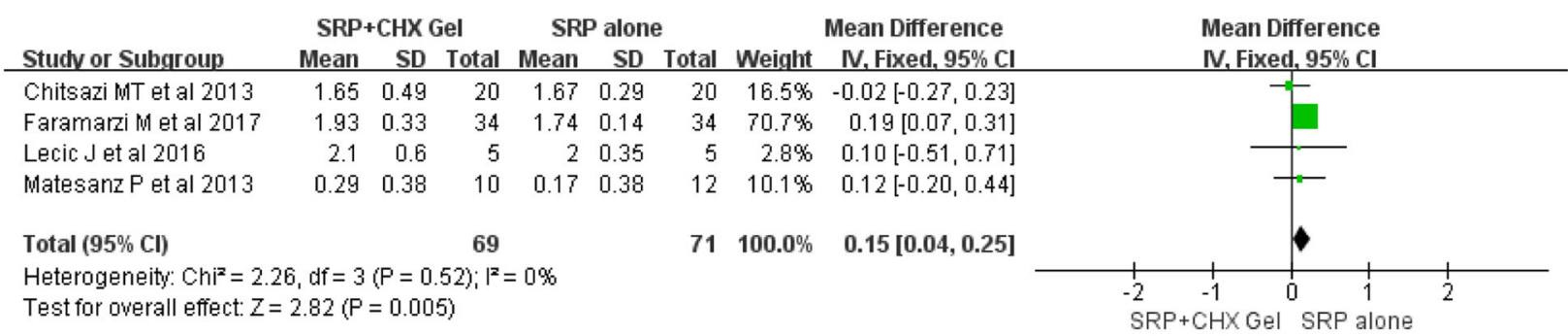

\begin{tabular}{|c|c|c|c|c|c|c|c|c|c|c|c|c|}
\hline \multirow[b]{2}{*}{ Studv or Subgroup } & \multicolumn{3}{|c|}{ SRP+CHX Gel } & \multicolumn{3}{|c|}{ SRP alone } & \multicolumn{3}{|c|}{ Mean Difference } & \multirow{2}{*}{\multicolumn{3}{|c|}{$\begin{array}{c}\text { Mean Difference } \\
\text { IV, Random, } 95 \% \mathrm{Cl}\end{array}$}} \\
\hline & Mean & SD & Total & Mean & SD & Total & Weight & IV, Random, 95\% Cl & Year & & & \\
\hline Chitsazi MT et al 2013 & 0.47 & 0.23 & 20 & 0.5 & 0.15 & 20 & $36.4 \%$ & $-0.03[-0.15,0.09]$ & 2013 & & & \\
\hline Matesanz $P$ et al 2013 & 0.16 & 0.47 & 10 & 0.14 & 0.45 & 12 & $8.4 \%$ & $0.02[-0.37,0.41]$ & 2013 & & & \\
\hline Lecic J et al 2016 & 0.35 & 0.51 & 5 & 0.85 & 0.64 & 5 & $2.7 \%$ & $-0.50[-1.22,0.22]$ & 2016 & & & \\
\hline Faramarzi M et al 2017 & 0.87 & 0.1 & 34 & 0.77 & 0.09 & 34 & $52.5 \%$ & $0.10[0.05,0.15]$ & 2017 & & 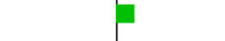 & \\
\hline Total $(95 \% \mathrm{Cl})$ & & & 69 & & & 71 & $100.0 \%$ & $0.03[-0.09,0.15]$ & & & & \\
\hline \multicolumn{7}{|c|}{$\begin{array}{l}\text { Heterogeneity: } \text { Tau }^{2}=0.01 ; \mathrm{Chi}^{2}=6.56, \mathrm{df}=3(\mathrm{P}=0.09) ; \mathrm{I}^{2}=54 \% \\
\text { Test for overall effect: } \mathrm{Z}=0.48(\mathrm{P}=0.63)\end{array}$} & & & & -2 & $\begin{array}{lll}-1 & 0 & 1 \\
+\mathrm{CH} & 0 & -1 \\
& \mathrm{SRP} \text { alon }\end{array}$ & 2 \\
\hline
\end{tabular}

Fig. 2 Forest plots comparing the adjunctive use of chlorhexidine (CHX) gel to scaling and root planing (SRP) and SRP alone at selected sites at 3 months: a probing pocket depth (PPD) reduction; b clinical attachment level (CAL) gain 


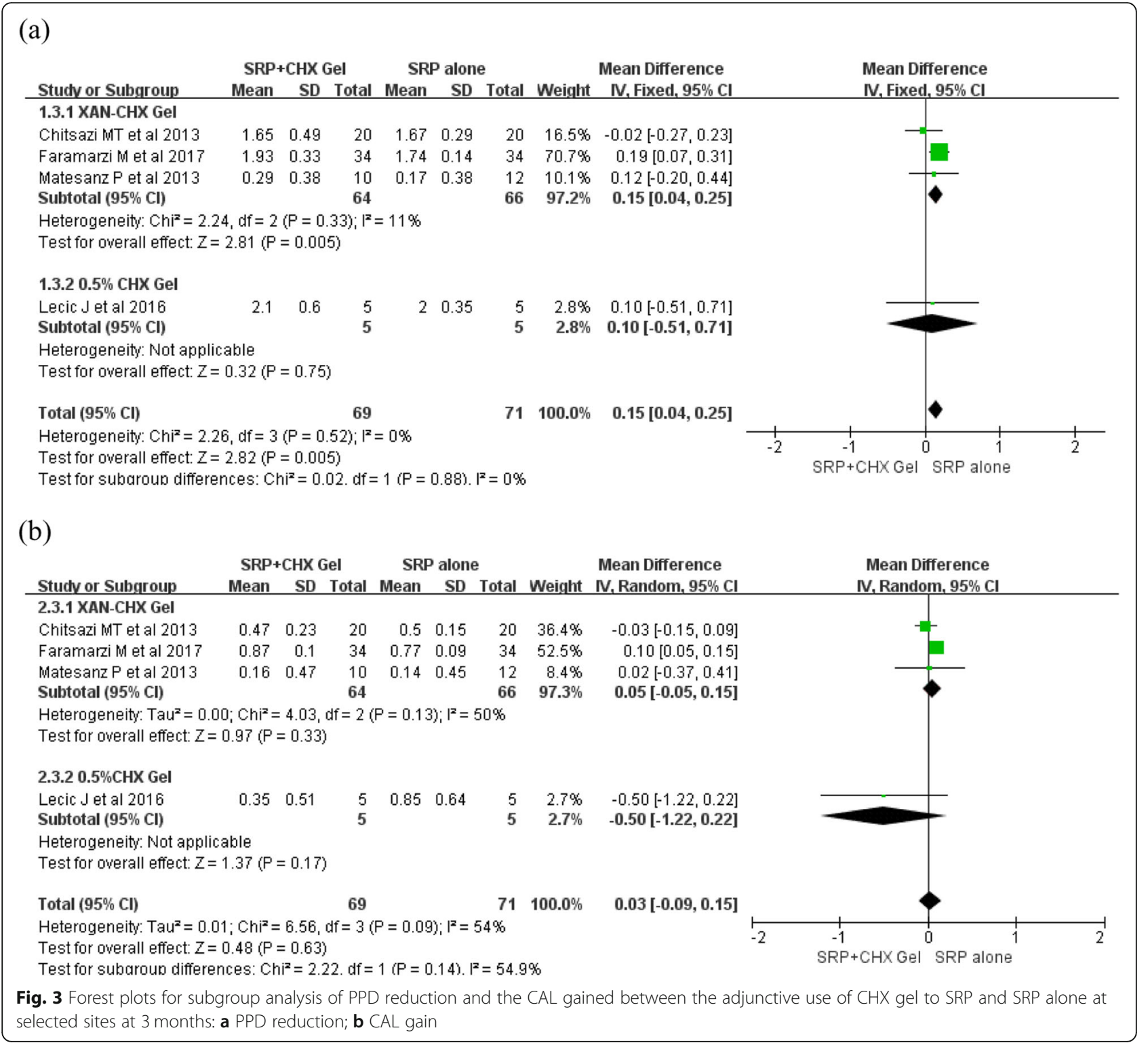

3-4 months and $0.05 \mathrm{~mm}$ at $6-8$ months with no heterogeneity [3-4 months (MD: 0.09 [95\% CI: - 0.27-0.46]; $p=0.61, \mathrm{I} 2=0 \%$ ) (Fig. 5a), 6-8 months (MD: 0.05 [95\% CI: $-0.29-0.39$ ]; $p=0.78, \mathrm{I} 2=0 \%$ ) (Fig. 5b)].

\section{Adverse events}

Four studies reported adverse effects after treatment [17, $18,28,31]$. Only one study comparing FMD and FMSRP reported that 17 subjects in the FMD and 12 in the FMSRP groups had one or two adverse events following mouth rinses, including changes in taste perception, dry mouth and staining [17].

\section{Discussion}

Four trials comparing adjunctive $\mathrm{CHX}$ gel and SRP with SRP alone at selected sites were included for quantitative analysis. The results showed that adjunctive administration of CHX gel provided a significant improvement in PPD reduction with a small overall effect size of 0.15 $\mathrm{mm}$ and no benefit to CAL. For subgroup analysis, adjunctive subgingival administration of XAN-CHX gel containing 1.5\% $\mathrm{CHX}$ provided also a slightly greater improvement of PPD reduction of MD $0.15 \mathrm{~mm}$.

In qualitative analysis, $\mathrm{CHX}$ gel without Xanthan gum was applied as adjunct to SRP at selected sites in three studies, and showed no beneficial clinical outcomes [1012]. The results were consistent with various studies reporting minimal benefits in the local use of traditional CHX gel as a monotherapy $[36,37]$ or as an adjunct to SRP [10-13]. The effect of locally delivered antimicrobial drugs depends on its concentration and contact time in the local environment [12]. For subgingival 


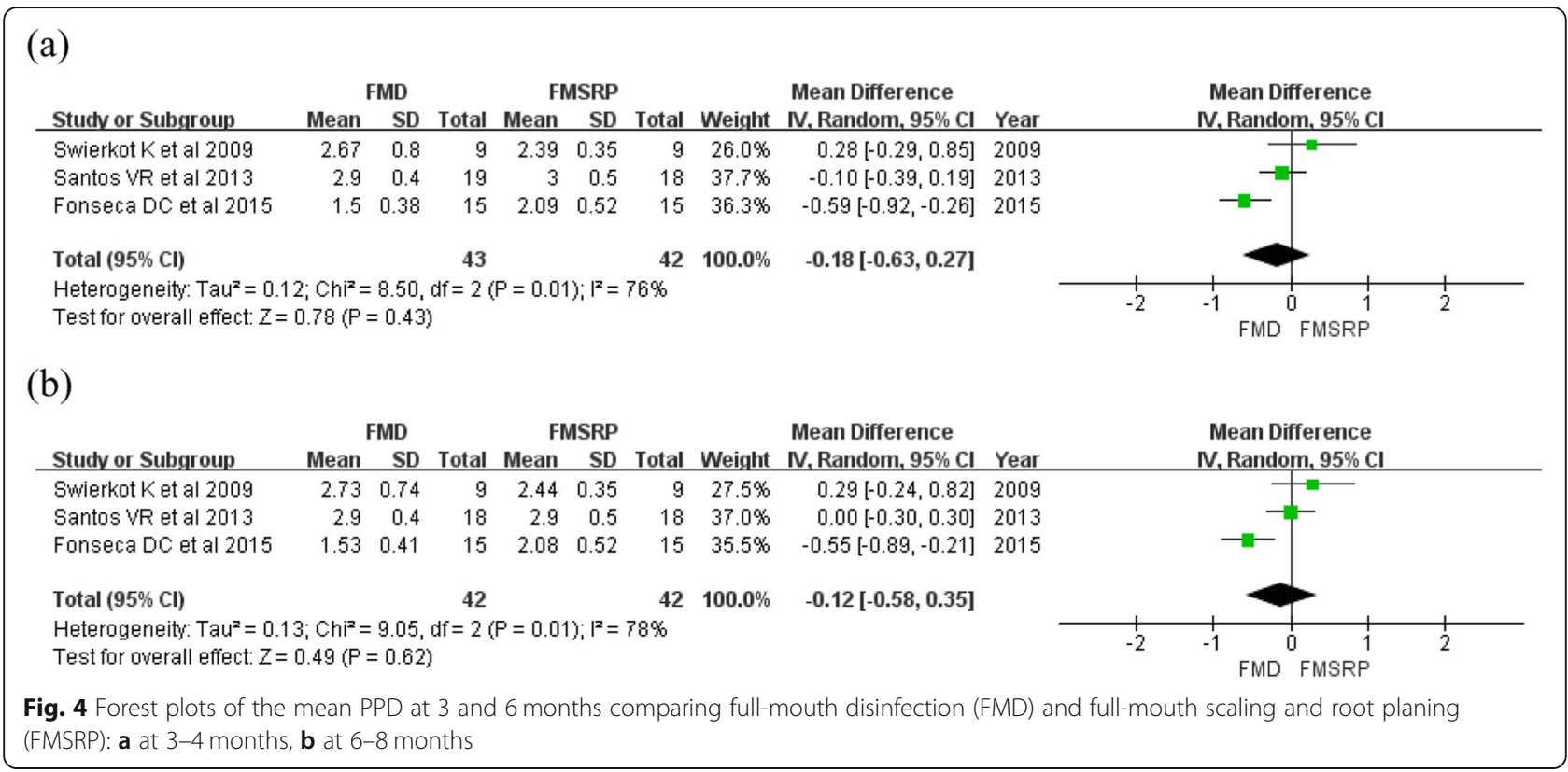

administration of drugs, the outflow of crevicular fluid may play an important role. Evidence indicated that the outflow of crevicular fluid is about $20 \mathrm{ml} /$ hour, which might be the main cause of the short-term half-life of the gel within the periodontal pocket $[14,38]$. Oosterwaal et al. applied fluorescein gel in four pockets of 10 patients, samples were taken from 1 of the 4 pockets at 5, 10, 20 and $40 \mathrm{~min}$. The results showed that the most locally delivered gels in the pocket disappeared within 5 min after application, which might be due to the elasticity of pocket soft tissue, bleeding after drug administration and spreading of the gel. And then the gel was washed out linearly and gradually by crevicular fluid flow and released from the adherent surface of the periodontal pocket [14]. Given the high clearance of $\mathrm{CHX}$ within the pockets, $\mathrm{CHX}$ gel seemed to not be an effective adjuvant to SRP. XAN-CHX gel has been applied for local periodontal treatment within the recent 10 years, which contains a mixture of CHX digluconate and CHX dihydrochloride, incorporated in a Xanthan gum. XAN-CHX gel demonstrated a greater capacity to increase viscosity of the carrier (CHX) and maintained

\section{(a)}

\begin{tabular}{|c|c|c|c|c|c|c|c|c|c|c|c|c|}
\hline \multirow[b]{2}{*}{ Study or Subgroup } & \multicolumn{3}{|c|}{ FMD } & \multicolumn{3}{|c|}{ FMDSRP } & \multicolumn{3}{|c|}{ Mean Difference } & \multirow{2}{*}{\multicolumn{2}{|c|}{$\begin{array}{l}\text { Mean Difference } \\
\text { IV, Fixed, 95\% Cl }\end{array}$}} & \\
\hline & Mean & SD & Total & Mean & SD & Total & Weight & IV, Fixed, $95 \% \mathrm{Cl}$ & Year & & & \\
\hline Swierkot Ket al 2009 & 3.37 & 0.67 & 9 & 3.13 & 1.09 & 9 & $18.9 \%$ & $0.24[-0.60,1.08]$ & 2009 & & & \\
\hline Santos VR et al 2013 & 3.7 & 0.92 & 19 & 3.9 & 0.87 & 18 & $39.7 \%$ & $-0.20[-0.78,0.38]$ & 2013 & & - & \\
\hline Fonseca DC et al 2015 & 2.48 & 0.81 & 15 & 2.17 & 0.77 & 15 & $41.3 \%$ & $0.31[-0.26,0.88]$ & 2015 & & & \\
\hline Total $(95 \% \mathrm{Cl})$ & & & 43 & & & 42 & $100.0 \%$ & $0.09[-0.27,0.46]$ & & & & \\
\hline $\begin{array}{l}\text { Heterogeneity: Chi }=1.6 \\
\text { Test for owerall effect: } Z=\end{array}$ & $\begin{array}{l}\mathrm{df}=2 \\
51(\mathrm{P}\end{array}$ & $\begin{array}{l}P=0 . \\
=0.61)\end{array}$ & $43) ; 1^{2}=$ & $0 \%$ & & & & & & -2 & $\begin{array}{lll}-1 & 0 & 1 \\
& \text { FMD FMSRP }\end{array}$ & 2 \\
\hline
\end{tabular}

(b)

\begin{tabular}{|c|c|c|c|c|c|c|c|c|c|c|c|c|}
\hline \multirow[b]{2}{*}{ Studv or Subgroup } & \multicolumn{3}{|c|}{ FMD } & \multicolumn{3}{|c|}{ FMSRP } & \multicolumn{3}{|c|}{ Mean Difference } & & \multirow{2}{*}{$\begin{array}{l}\text { Mean Difference } \\
\text { IV. Fixed, 95\% Cl }\end{array}$} & \\
\hline & Mean & SD & Total & Mean & SD & Total & Weight & IV, Fixed, $95 \% \mathrm{CI}$ & Year & & & \\
\hline Swierkot Ket al 2009 & 3.35 & 0.67 & 9 & 3.15 & 0.85 & 9 & $23.2 \%$ & $0.20[-0.51,0.91]$ & 2009 & & & \\
\hline Santos VR et al 2013 & 3.6 & 0.77 & 19 & 3.8 & 0.92 & 18 & $38.6 \%$ & $-0.20[-0.75,0.35]$ & 2013 & & & \\
\hline Fonseca DC et al 2015 & 2.41 & 0.8 & 15 & 2.2 & 0.74 & 15 & $38.2 \%$ & $0.21[-0.34,0.76]$ & 2015 & & & \\
\hline Total $(95 \% \mathrm{Cl})$ & & & 43 & & & 42 & $100.0 \%$ & $0.05[-0.29,0.39]$ & & & & \\
\hline \multicolumn{13}{|c|}{ 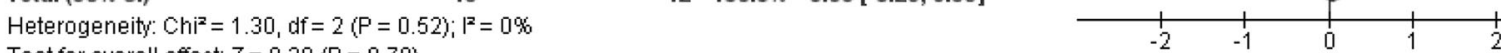 } \\
\hline
\end{tabular}

Fig. 5 Forest plots of the mean CAL at 3 and 6 months comparing full-mouth disinfection (FMD) and full-mouth scaling and root planing (FMSRP): $\mathbf{a}$ at $3-4$ months, $\mathbf{b}$ at $6-8$ months 
the bacteriostatic and bactericidal concentrations for at least 2 weeks inside the periodontal pocket [35], which could further promote its pharmacotherapeutic effects. Based on this evidence, XAN-CHX gel may overcome the limitations of the previously used $\mathrm{CHX}$ gel. Considering the results of this meta-analysis for subgroups, XAN-CHX gel provided only a minor additional improvement with mean MD of $0.15 \mathrm{~mm}$ of PPD reduction, and no benefit of CAL gain. So far, no sufficient data have supported the clinical efficacy of adjunctive subgingival applications of XAN-CHX gel according to existing research. Evidence has reported that CHX has a high affinity for salivary or serum proteins and blood, which might lead to its rapid concentration decrease in the subgingival environment [39-41]. Furthermore, the behaviour of pathogenic bacteria in periodontal pocket may also resist the effectiveness of local CHX gel. Evidence supported that P.g releases vesicles capable of inactivating the CHX molecule, thereby protecting themselves and other bacteria from the bactericidal activity [15]. These features may markedly negatively regulate the effects of subgingival administrations of a XAN-CHX gel. Microbiological outcomes of various studies have confirmed the minimal efficacy of locally delivered XAN-CHX gel as an adjunct to SRP, which showed minor bacterial count reductions in an adjunctive XANCHX gel group as compared to control [29, 31, 34, 42]. Due to a lack of microbiological data from consistent testing methods and standards, microbiological outcomes were not analysed in the review.

In recent years, $\mathrm{CHX}$ gel has been commonly used for FMD protocol in the treatment of periodontal disease. Considering the clinical benefit of FMD in varying degrees, FMD protocol has been conducted in a large number of studies [43-46]. In addition to full-mouth subgingival applications of $\mathrm{CHX}$ gel, tongue brush with CHX gel and mouthwash with CHX solution were also performed with the aim of maximum elimination of periodontal pathogens in the mouth. Despite these, no additional benefits for the adjunctive use of CHX in FMSRP were shown in this meta-analysis. This result is consistent with other studies and reviews, which indicated that the benefits of FMD probably resulted from the short-term full-mouth mechanical debridement, rather than the beneficial effects of CHX [15, 47]. A high heterogeneity was detected for analysis of the mean PPD between the FMD and FMSRP groups at 3 months (I2 = $76 \%)$ and 6 months (I2 $=78 \%)$. Regarding the small number of included studies and limited data available, there were variable factors impacting on the results, such as the general health of the included patients, the initial disease severity of the chronic periodontitis, the frequency of $\mathrm{CHX}$ gel application and the influence of other adjunctive means of CHX included in FMD and its period and frequency.
Noticeably, side effects and adverse events related to the use of the local administration of CHX in the treatment of periodontitis should be taken into account and be weighed against the potential benefits. Although the local application of antiseptics or antibiotics overcomes uncertainties in the systemic use of antibiotics, adverse events, such as changes in taste perception, dry mouth, erythema, oral ulceration, gingival tingling, periodontal abscesses, root sensitivity and staining of tongue or teeth, were reported [48]. For FMD, due to long-term mouthwash using $\mathrm{CHX}$ solution, staining could occur in most patients [18]. This fact should remind clinicians that the balance between the small effect size of clinical benefit and high possibility of tooth staining should be taken in consideration when developing a treatment plan for periodontal patients.

\section{Limitations}

To the best of our knowledge, this is the first systematic review focusing on the effects of adjunctive subgingival application of CHX gel to SRP. Therefore, we cannot compare our results with previous publications. In addition, because of the lack of RCTs with high quality, only seven studies with a small number of participants were included for quantitative meta-analysis, and longterm clinical results comparing the test and control groups were not calculated. More RCTs with more participants and long-term follow-up are needed in the future.

\section{Conclusion}

Based on the results of this meta-analysis, adjunctive subgingival administration of XAN-CHX gel at individual selected sites with PPD at least $4 \mathrm{~mm}$ promotes a slight additional benefit in PPD reduction. Adjunctive antiseptics of CHX gel at specific sites might be advisable, but SRP always plays the dominate role in the treatment of chronic periodontitis. Due to a lack of high-quality studies, more RCTs with larger sample sizes and strict standards are needed to confirm these conclusions.

\section{Supplementary information}

Supplementary information accompanies this paper at https://doi.org/10. 1186/s12903-020-1021-0.

Additional file 1: Table S1. Reasons for exclusion of studies.

Additional file 2: Table S2. Summary of clinical outcomes.

\section{Abbreviations}

CAL: Clinical attachment levels; CHX: Chlorhexidine; CP: Chronic periodontitis; FMD: Full-mouth disinfection; FMSRP: Full-mouth scaling and root planning; NSPT: Nonsurgical periodontal treatment; PPD: Probing pocket depth; SRP: Scaling and root planing; XAN-CHX: Xanthan-based chlorhexidine 


\section{Acknowledgements}

The authors would like to thank the support of China Scholarship Council and Beijing Stomatological Hospital, School of Stomatology, Capital Medical University for this study.

\section{Authors' contributions}

$\mathrm{HZ}$ and $\mathrm{JCH}$ contributed to study conception and design. $\mathrm{HZ}$ collected the data and informations, and drafted the manuscript; JCH critically revised the manuscript; LZ performed the statistical analysis. All authors gave final approval and agree to be accountable for all aspects of the work.

\section{Funding}

JCH was supported by the China Scholarship Council for research abroad in University Witten/Herdecke. HZ was supported by Beijing Stomatological Hospital, School of Stomatology, Capital Medical University.

\section{Availability of data and materials}

The datasets used and/or analysed during the current study are available from the corresponding author on reasonable request.

\section{Ethics approval and consent to participate}

Not applicable.

\section{Consent for publication}

Not applicable.

\section{Competing interests}

The authors declare that they have no competing interests.

\section{Author details}

'Multi-disciplinary Treatment Center, Beijing Stomatological Hospital, School of Stomatology, Capital Medical University, Tian Tan Xi Li Number.4, Beijing 100050, China. ${ }^{2}$ Department of Periodontology, Witten/Herdecke, University, Alfred-Herrhausen-Str. 45, 58445 Witten, Germany. ${ }^{3}$ Department of Periodontics, Beijing Stomatological Hospital, School of Stomatology, Capital Medical University, Tian Tan Xi Li Number 4, Beijing 100050, China. ${ }^{4}$ Department of Prosthodontics, Stomatological Hospital of Chongqing Medical University, Chongqing 400015, China. ${ }^{5}$ Chongqing Key Laboratory of Oral Diseases and Biomedical Sciences, Chongqing 400015, China. ${ }^{6}$ Chongqing Municipal Key Laboratory of Oral Biomedical Engineering of Higher Education, Chongqing 400015, China.

\section{Received: 28 October 2019 Accepted: 23 January 2020}

\section{Published online: 31 January 2020}

\section{References}

1. Quirynen M, Teughels W, De Soete M, van Steenberghe D. Topical antiseptics and antibiotics in the initial therapy of chronic adult periodontitis: microbiological aspects. Periodontol 2000. 2002;28:72-90.

2. Quirynen M, De Soete M, Boschmans G, Pauwels M, Coucke W, Teughels W, et al. Benefit of "one-stage full-mouth disinfection" is explained by disinfection and root planing within 24 hours: a randomized controlled trial. J Clin Periodontol. 2006;33:639-47.

3. Renvert S, Wikström M, Dahle'n G, Slots J, Egelberg J. Effect of root debridement on the elimination of Actinobacillus actinomycetemcomitans and Bacteroides gingivalis from periodontal pockets. J Clin Periodontol. 1990; 17:345-50.

4. Renvert S, Dahle'n G, Wikström M. The clinical and microbiological effects of non-surgical periodontal therapy in smokers and non-smokers. J Clin Periodontol. 1998;25:153-7.

5. Mombelli A, Gmür R, Gobbi C, Lang NP. Actinobacillus actinomycetemcomitans in adult periodontitis. II. Characterization of isolated strains and effect of mechanical periodontal treatment. J Periodontol. 1994;65:827-34.

6. Mombelli A, Schmid B, Rutar A, Lang NP. Persistence patterns of Porphyromonas gingivalis, Prevotella intermedia/nigrescens, and Actinobacillus actinomyetemcomitans after mechanical therapy of periodontal disease. J Periodontol. 2000;71:14-21.

7. Akram Z, Vohra F, Javed F. Locally delivered metformin as adjunct to scaling and root planing in the treatment of periodontal defects: a systematic review and meta-analysis. J Periodontal Res. 2018;53:941-9.
8. Castro MML, Duarte NN, Nascimento PC, Magno MB, Fagundes NCF, FloresMir $\mathrm{C}$, et al. Antioxidants as adjuvants in periodontitis treatment: a systematic review and meta-analysis. Oxidative Med Cell Longev. 2019;22: 9187978 eCollection 2019.

9. Azaripour A, Dittrich S, Van Noorden CJF, Willershausen B. Efficacy of photodynamic therapy as adjunct treatment of chronic periodontitis: a systematic review and meta-analysis. Lasers Med Sci. 2018;33:407-23.

10. Lecic J, Cakic S, Janjic Pavlovic O, Cicmil A, Vukotic O, Petrovic V, et al. Different methods for subgingival application of chlorhexidine in the treatment of patients with chronic periodontitis. Acta Odontol Scand. 2016;74:502-7.

11. Unsal E, Akkaya M, Walsh TF. Influence of a single application of subgingival chlorhexidine gel or tetra-cycline paste on the clinical parameters of adult periodontitis patients. J Clin Periodontol. 1994;21:351-5.

12. Oosterwaal PJ, Mikx FH, van't Hof MA, Renggli HH. Comparison of the antimicrobial effect of the application of chlorhexidine gel, amine fluoride gel and stannous fluoride gel in debrided periodontal pockets. J Clin Periodontol. 1991;18:245-51.

13. Vinholis AH, Figueiredo LC, Marcantonio Júnior E, Marcantonio RA, Salvador SL, Goissis G. Subgingival utilization of a 1\% chlorhexidine collagen gel for the treatment of periodontal pockets. A clinical and microbiological study. Braz Dent J. 2001;12:209-13.

14. Oosterwaal PJ, Mikx FH, Renggli HH. Clearance of a topically applied fluorescein gel from periodontal pockets. J Clin Periodontol. 1990;17:613-5.

15. Cosyn J, Sabzevar MM. A systematic review on the effects of subgingival chlorhexidine gel administration in the treatment of chronic periodontitis. J Periodontol. 2005;76:1805-13.

16. Quirynen M, Bollen CML, Vandekerckhove BNA, Dekeyser C, Papaioannou W, Eyssen H. Full- vs. partial-mouth disinfection in the treatment of periodontal infections: short-term clinical and microbiological observations. J Dent Res. 1995;74:1459-67.

17. Fonseca DC, Cortelli JR, Cortelli SC, Miranda Cota LO, Machado Costa LC, Moreira Castro MV, et al. Clinical and microbiologic evaluation of scaling and root Planing per quadrant and one-stage full-mouth disinfection associated with azithromycin or Chlorhexidine: a clinical randomized controlled trial. J Periodontol. 2015;86:1340-51.

18. Santos VR, Lima JA, Miranda TS, Gonçalves TE, Fiqueiredo LC, Faveri M, et al. Full-mouth disinfection as a therapeutic protocol for type-2 diabetic subjects with chronic periodontitis: twelve-month clinical outcomes: a randomized controlled clinical trial. J Clin Periodontol. 2013;40:155-62.

19. Swierkot K, Nonnenmacher Cl, Mutters R, Flores-de-Jacoby L, Mengel R. One-stage full-mouth disinfection versus quadrant and full-mouth root planing. J Clin Periodontol. 2009:36:240-9.

20. Lang NP, Tan WC, Krähenmann MA, Zwahlen M. A systematic review of the effects of full-mouth debridement with and without antiseptics in patients with chronic periodontitis. J Clin Periodontol. 2008;35(Suppl 8):8-21.

21. Moher D, Altman DG, Liberati A, Tetzlaff J. PRISMA statement. Epidemiology. 2011:22:128

22. Mchugh ML. Interrater reliability: the kappa statistic. Biochem Med (Zagreb). 2012;22(3):276-82

23. Moher D, Schulz KF, Altman DG, CONSORT GROUP (consolidated standards of reporting trials). The CONSORT statement: Revised recommendations for improving the quality of reports of parallel-group randomized trials. Ann Intern Med. 2001;134:657-62.

24. Higgins JPT, Altman DG, JAC S. Chapter 8: Assessing risk of bias in included studies. In: JPT H, Churchill R, Chandler J, Cumpston MS, editors. Cochrane Handbook for Systematic Reviews of Interventions version 5.2.0 (updated June 2017); 2017. p. 1-73.

25. Higgins JPT, Thompson SG, Deeks JJ, Altman DG. Measuring inconsistency in meta-analyses. BMJ. 2003;327:557-60.

26. Faramarzi M, Shirmohammadi A, Chitsazi M, Shamami MS, Ghanitab S Clinical and metabolic effects of subgingival application of xanthan-based chlorhexidine gel in type 2 diabetic patients with chronic periodontitis. Dent Res J (Isfahan). 2017;14:299-305.

27. Phogat M, Rana T, Prasad N, Baiju CS. Comparative evaluation of subgingivally delivered xanthan-based chlorhexidine gel and herbal extract gel in the treatment of chronic periodontitis. J Indian Soc Periodontol. 2014; 18:172-7.

28. Jain M, Dave D, Jain P, Manohar B, Yadav B, Shetty N. Efficacy of xanthan based chlorhexidine gel as an adjunct to scaling and root planing in treatment of the chronic periodontitis. J Indian Soc Periodontol. 2013;17: 439-43. 
29. Chitsazi MT, Kashefimehr A, Pourabbas R, Shirmohammadi A, Ghasemi Barghi V, Daghigh AB. Efficacy of subgingival application of xanthan-based Chlorhexidine gel adjunctive to full-mouth root Planing assessed by realtime PCR: a microbiologic and clinical study. J Dent Res Dent Clin Dent Prospects. 2013;7:95-101.

30. Chauhan AS, Bains VK, Gupta V, Singh GP, Patil SS. Comparative analysis of hyaluronan gel and xanthan-based chlorhexidine gel, as adjunct to scaling and root planing with scaling and root planing alone in the treatment of chronic periodontitis: a preliminary study. Contemp Clin Dent. 2013;4:54-61.

31. Matesanz P, Herrera D, Echeverría A, O'Connor A, Gon-zález I, Sanz M. A randomized clinical trial on the clinical and microbiological efficacy of a xanthan gel with chlor-hexidine for subgingival use. Clin Oral Invest. 2013;17:55-66.

32. Verma A, Sanghi S, Grover D, Aggarwal S, Gupta R, Pandit N. Effect of insertion of xanthan-based chlorhexidine gel in the maintenance phase following the treatment of chronic periodontitis. J Indian Soc Periodontol. 2012:16:381-5.

33. Kranti K, Seshan H, Sameer Z. Clinical evaluation of topical subgingival application of biodegradable xanthan based 1.5\% chlorhexidine gel for treatment on periodontal pockets. J Adv Dent Res. 2010;1:47-54.

34. Paolantonio M, D'Ercole S, Pilloni A, D'Archivio D, Lisanti L, Graziani F, et al. Clinical, microbiologic, and biochemical effects of subgingival administration of a xanthan-based chlorhexidine gel in the treatment of periodontitis: a randomized multicenter trial. J Periodontol. 2009;80:1479-92.

35. Gupta R, Pandit N, Aggarwal S, Verma A. Comparative evaluation of subgingivally delivered 10\% doxycycline hyclate and xanthan-based chlorhexidine gels in the treatment of chronic periodontitis. J Contemp Dent Pract. 2008;9:25-32.

36. Oosterwaal PJ, Mikx FH, van't Hof MA, Renggli HH. Short-term bactericidal activity of chlorhexidine gel, stannous fluoride gel and amine fluoride gel tested in periodontal pockets. J Clin Periodontol. 1991;18:97-100.

37. Perinetti G, Paolantonio M, Cordella C, D'Ercole S, Serra E, Piccolomini R. Clinical and microbiological effects of subgingival administration of two active gels on persistent pockets of chronic periodontitis patients. J Clin Periodontol. 2004;31:273-81.

38. Goodson J. Pharmacokinetic principles controlling efficacy of oral therapy. J Dent Res. 1989;68:1625-32.

39. Wade WG, Addy M. In vitro activity of a chlorhexidine containing mouthwash against subgingival bacteria. J Periodontol. 1989;60:521-5.

40. Rabe LK, Hillier SL. Effect of chlorhexidine on genital microflora, Neisseria gonorrhoeae, and Trichomonas vaginalis in vitro. Sex Transm Dis. 2000;27:74-8.

41. Slots J. Selection of antimicrobial agents in periodontal therapy. J Periodontal Res. 2002;37:389-98.

42. Manthena S, Ramesh A, Srikanth A, Ramoji Rao MV, Preethi PL, Samatha YP. Comparative evaluation of subgingivally delivered chlorhexidine varnish and chlorhexidine gel in reducing microbial count after mechanical periodontal therapy. J Basic Clin Pharm. 2014;6:24-8.

43. Boia S, Stratul S-ı, Boariu M, Ursoniu S, Goția SL, Boia ER, et al. Evaluation of antioxidant capacity and clinical assessment of patients with chronic periodontitis treated with non-surgical periodontal therapy and adjunctive systemic antibiotherapy. Romanian J Morphol Embryol. 2018;59:1107-13.

44. Almeida ML, Duarte PM, Figueira EA, et al. Effects of a full-mouth disinfection protocol on the treatment of type-2 diabetic and non-diabetic subjects with mild-to-moderate periodontitis: one-year clinical outcomes. Clin Oral Investig. 2019. https://doi.org/10.1007/s00784-019-02927-8.

45. Pockpa AD, Soueidan A, Louis P, Coulibaly NT, Badran Z, Struillou X. Twenty Years of Full-Mouth Disinfection: The Past, the Present and the Future. Open Dent J. 2018;12:435-42.

46. Santuchi CC, Cortelli JR, Cortelli SC, Cota LO, Fonseca DC, Alencar CO, et al. Scaling and root Planing per quadrant versus one-stage full-mouth disinfection: assessment of the impact of chronic periodontitis treatment on quality of life--a clinical randomized, controlled trial. J Periodontol. 2016;87:114-23.

47. Quirynen M, Mongardini C, De Soete M, Pauwels M, Coucke W, van Eldere J, et al. The role of chlorhexidine in the one-stage full-mouth disinfection treatment of patients with advanced adult periodontitis. Long-term clinical and microbiological observations. J Clin Periodontol. 2000;27:578-89.

48. Matesanz-Pérez P, García-Gargallo M, Figuero E, Bascones-Martínez A, Sanz $M$, Herrera D. A systematic review on the effects of local antimicrobials as adjuncts to subgingival debridement, compared with subgingival debridement alone, in the treatment of chronic periodontitis. J Clin Periodontol. 2013;40:227-41.

\section{Publisher's Note}

Springer Nature remains neutral with regard to jurisdictional claims in published maps and institutional affiliations.

Ready to submit your research? Choose BMC and benefit from:

- fast, convenient online submission

- thorough peer review by experienced researchers in your field

- rapid publication on acceptance

- support for research data, including large and complex data types

- gold Open Access which fosters wider collaboration and increased citations

- maximum visibility for your research: over $100 \mathrm{M}$ website views per year

At BMC, research is always in progress.

Learn more biomedcentral.com/submissions 